\title{
Malignant Lipomatous Neoplasm
}

National Cancer Institute

\section{Source}

National Cancer Institute. Malignant Lipomatous Neoplasm. NCI Thesaurus. Code C4501.

A malignant mesenchymal neoplasm arising from adipocytes. 